\title{
Issues and Challenges in Cloud Computing
}

\author{
Deepak Kumar Verma ${ }^{1}$, Tanya Sharma \\ Department of Computer Science, Babasaheb Bhimrao Ambedkar University (A Central University), Lucknow, India ${ }^{1,2}$
}

\begin{abstract}
Cloud computing is a model that helps to speed up and increase the flexibility of data management with reduced cost. It is undeniable that cloud computing has brings us lots of benefits and becoming more popular nowadays. Many large companies start using cloud service in their business. While the cloud computing is widely used, the security becomes a concern to everyone who use cloud services. There is a lot of security arises continuously while there are improvement as well on the security model of the cloud service provided. Despite the increasing use of the cloud service, the user should use the cloud service provided wisely in a way that always ensure good security practices so that this technology have the potential to bring the information technology to the next level. In this paper we have provided the foundation in regards to major issues and challenges in cloud computing and we have also proposed a data recovery technique in cloud computing.
\end{abstract}

Keywords: Cloud Computing, Issues and Challenges, Security, Cloud Models

\section{INTRODUCTION}

Like real clouds which are the collection of water molecules, the term "cloud" in cloud computing is the collection of networks. The user can use the modalities of cloud computing boundlessly whenever demanded. Instead of setting up their own physical infrastructure, the users ordinarily prefer a mediator provider for the service of the internet in cloud computing. The users have to pay only for the services they had used. The workload can be shifted to reduce the workload in cloud computing. A load of service is handled by the networks which forms the cloud that's why the load on local computers is not heavy while running an application. So the requisition of hardware and software at the user side is decreased. All we need to have a web browser to use cloud computing. All we need to have a web browser like chrome to use cloud computing.[1] Cloud is an extend of grid computing, distributed computing and parallel computing where everything is serve as service. It is a model for enabling configurable computing resources. Thus cloud computing can be said convenient, on demand network access to a shared pool of to provide Internet-based computing service. Cloud computing builds on already existing computing technologies, such as, grid computing and virtualization. These are the forms of the distributed computing technologies. [2] Cloud computing assign remote services with a user's data, computation and software. It is a model which provide on demand access to a shared pool computing resource like servers, storage, networks, applications and services. Cloud computing and storage solutions provide users and enterprises with various capabilities to store and process their data in third-party data centre's. It depends on sharing of resources to acquire coherence and economy of scale over a network. Due to the advantages like cheap cost of services, high performance high computing power, scalability, accessibility and availability cloud computing has become demanded.

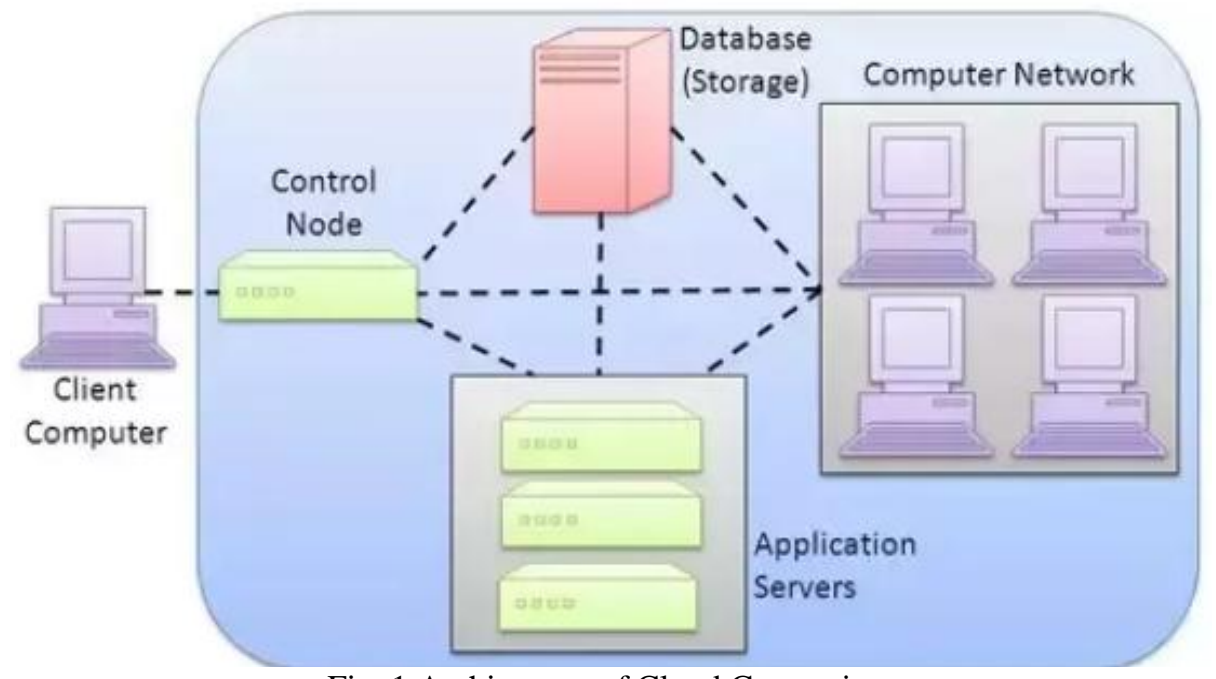

Fig. 1 Architecture of Cloud Computing 
Cloud computing takes the emphasis away from local computers. It is less about the machine you use at home or on the move and more about what is happening on computers many miles away. Instead of having to store information on your PC, smartphone or tablet, your data can be kept remotely. It will then be made available to any device that is capable of reading it. Typically, most cloud computing is conducted via a web browser but there are other ways, including specially-made apps for computers, tablets and smartphones. These act as access points for cloud services. They also mean that you don't always need to have dedicated software installed on your machines. The benefit of this is clear: suddenly you are not tied to your own computer. You do not even need to be tied to your own phone. All you need is a way of accessing the data and that be done from any machine. As you can see, this differs from traditional computing because you do not need portable storage media nor are you relying on how robust or fast your machine is. With cloud computing, you are seeing programs and data being managed and provided as a service over the internet and it opens up many possibilities.

\section{REVIEW OF LITERATURE}

Prof. Meera Randiva [1] ,have discussed important to find out an alternate source for the storage of their data.at the same time, this storage should be safe, easy to handle and economical. Moreover, it would provide storage according to the demand and paid accordingly. Therefore, cloud computing has become a vital part for all the private sectors as well as government sectors in order to fulfill their demand and Many ITs companies has large demand of data storage, which is not possible on their parts.

Mr. PathanNouman Khan[2] , In this paper author have discussed Internet based computing which provides you shared processing resources and data to systems and other devices on respective demand. Central remote servers and Internet are used to maintain application and data in cloud computing. It allows using application without access and installation their personal files on computer with internet access because of which data storage, bandwidth and processing became more efficient.

Mahantesh Birje [5], In this paper author have discussed Cloud computing delivers IT-related capabilities as a service through internet to multiple customers and these services are charged based on consumption. Many cloud computing providers such as Google, Microsoft, Yahoo, IBM and Amazon are moving towards adoption of cloud technology leading to considerable escalation in the usage of various cloud services. This paper reviews cloud computing paradigm in terms of its historical evolution, concepts, technology, tools and various challenges. This review paper helps researchers who would like to begin their research career in cloud computing area.

NasarulIslam.K.V [6], discussed that this technology has numerous advantages, even though it has not satisfied its maturity level due to the lack of security and other issues. This paper deals with the various advantages and security challenges of cloud computing. It also point out the various service models of cloud computing. Author says Cloud computing is fastest booming technology; nowadays it has remarkable position in business concern. This technology has changed the face of traditional computing technologies, and simplest service available through internet to start and maintain business.

Sanjana Sharma, SonikaSoni, Swati Sengar[7], In this paper gives a brief introduction of cloud computing its types and security issue and approaches to secure the data in the cloud environment. Cloud computing is a long dreamed vision of computing as a utility, where data owners can remotely store their data in the cloud to enjoy on- demand highly-quality application and services from a shared pool of configurable computing resources.

Levent Ertau [8], In this paper author discussed investigates few major security issues with cloud computing and the existing counter measures to those security challenges in the world of cloud computing.. Security is as much of an issue in the cloud as it is anywhere else. Different people share different point of view on cloud computing. Some believe it is unsafe to use cloud. Cloud vendors go out of their way to ensure security. Document is a template. An electronic copy can be downloaded from the conference website. For questions on paper guidelines, please contact the conference publications committee as indicated on the conference website. Information about final paper submission is available from the conference website.

\section{SERVICE MODELS OF CLOUD COMPUTING}

\section{A. Infrastructure as a Service (IaaS)}

This service model delivers computer infrastructure as a service. This service is made available as a platform for virtualized machines. Unlike, traditional hardware machines which require special maintenance and limited flexibility, cloud makes these machines easily available virtually on the internet with flexible specifications and improved 
performance, optimized according to the requirements of the customer. Developers can run the platforms necessary for their software development and run them. This service also makes it easy for the customer to create instance for his required virtual machine simple and easy. In most of the cloud services provided by various service providers, setting up of virtual machines can be done with no or less cost. Cloud provides this virtualization feature in the form of containers. A direct virtual machine needs a hypervisor on its hardware above the kernel for efficient virtualization whereas containerization doesn't need a hypervisor which saves the processor efficiency and improves its performance. And also, container size is flexible i.e., it can be changed dynamically, hence eliminates over-provisioning. Generally, these virtual machines are installed as a form disk images, object, load balancers or IP addresses which can be dynamically installed on the cloud and also ensure the security of the virtual machine by allotting the virtual instance with a unique host address each time installed. These virtual instances are pre-installed on large pools of equipment called data centers. These virtual machines are billed by the service providers on the utility computing basis [4]. IaaS is a collection of servers, storage, and networks. Virtualization is the backbone behind this model where resources like network, storage, virtualized servers, routers and so on are consumed by user through virtual desktop, provided by pc's. Many computing resources are provided by the IaaS in the form of storage, network, operating system, hardware, and storage devices on demand. IaaS users can access the services using a wide area network, such as the internet [3]. Examples: rackspace, VMWare, Joyent, Storage services provided by Amazon S3, and Computation services provided by Amazon EC2.

\section{B. Platform as a Service (PaaS)}

This service model delivers platforms for building and running web-based applications. It provides all the facilities required to support the complete software development life cycle. This service basically delivers a computing platform for the customer who includes operating system, programming platforms, web servers, databases etc. Since everything is run on internet, there is no need to worry about the infrastructure and minimum requirements for the platform. This model can hence eliminates the worry of incompatibility of software environment on the machine, since hardware specifications required by the platform are met by the cloud service provider directly, thus providing powerful and unlimited computing power. Anyone with an internet connection can now develop powerful and efficient applications without worrying about the infrastructural and cost issues. The traditional on premise models were expensive and complex, which required specific, set of hardware and software specifications. For every problem statement, there is a different business solution, which meant different set of hardware and software specifications. This situation used to force the developers to change the application every now and then. Enormous electricity power was also required to run the hardware. With the entry of PaaS model of cloud, application development became quick, cost effective and efficient. PaaS provides infrastructure along with the workflow facilities required for the software development. It also provides application services for the software development such as security, storage, database integration, instrumentation etc. Another characteristic of PaaS models is the integration of web and mobile applications and services with the databases using Simple Object Access Protocol. PaaS is a collection of runtime environments such as software and development tools hosted on the provider's servers. It acts as background that provides runtime environment, software deployment framework and component on pay to facilitate the direct deployment of application level assets or web applications. A development environment or platform is given to the consumers as a service in PaaS, upon which user can deploy their own software and coding. The customer has the liberty to construct his own applications that can run on the provider's infrastructure [3]. Examples: Amazon AWS, Rollbase, jelastic.com, force.com, GoogleApp Engine (GAE), Microsoft Azure, and LONG JUMP.

\section{Software as a Service (SaaS)}

This service model provides the access to the application services and databases. Cloud providers take care of the infrastructure and platforms required to run the software applications on the Internet. It is sometimes referred to as 'ondemand software', which can be used after paying the subscription fees. In this model, cloud users directly install the subscribed applications on the cloud and directly access the software from their cloud clients. The cloud users need not manage the necessary infrastructure or the platforms required to run the software application. Some of the SaaS applications are Customer Relationship Management (CRM), Enterprise Resource Planning (ERP), accounting and other business software, which are mainly non-core-competency software. Most of the companies today opt for SaaS solutions, which don't require the employee to know the infrastructure, background logics and platform details to run the application. Instead he can just install the application on the cloud and run the application as a browser based service on the Internet. The present day advancements in cloud make it easier for the customer to use these SaaS applications anywhere at any time. These applications can be used on a web browser or a program interface without having to manage the specifications of the software. These applications have limited user-specific configuration settings which abstract most of the complex background details, making it easier for the user to deploy these applications. SaaS is a collection of software or services or applications available on cloud that can be accessed by end users based on subscription. End users consume the software application services through this service delivery model directly over 
Vol. 8, Issue 4, April 2019

network according on demand basis. The way of carrying application as a service on the internet is known as software as a service. In place of installing the software on his computer, the user can simply access it via the internet [3]. Examples: Whatsapp, Facebook, Twitter, Google Docs andspreadsheets, salesforce.com, NETSUITE, and IBM LotusLive.

Table 1: - Comparisons of Service Models

\begin{tabular}{|r|r|r|}
\hline Service Model $\rightarrow$ & Iaas \\
[Host]
\end{tabular}

\section{TYPES OF CLOUD DEPLOYMENT MODELS}

\section{A. Public Cloud}

Public cloud is defined as computing services offered by third party providers over the public internet, making them available to anyone who wants to use or purchase them. They may be free or sold on demand, allowing customers to pay only per usages for the cpu cycles, storage, or bandwidth they consume. This cloud allows cloud environment as openly accessible to all users. Public clouds off premise in which various enterprises can be used to deliver the services to users by taking it from the third party[5]. Examples: Google drive, Sun Cloud, Google AppEngine, IBM's Blue Cloud.

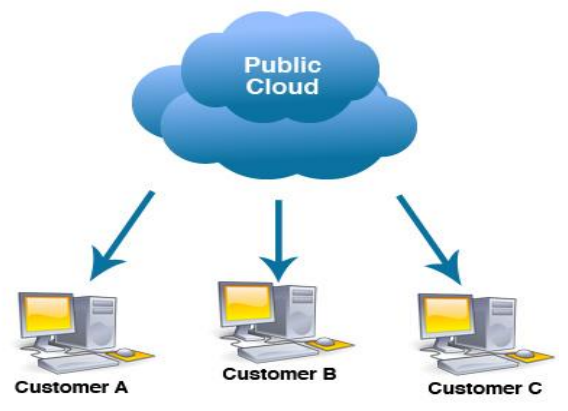

\section{B. Private Cloud}

A private cloud is a particular model of cloud computing that involves a distinct and secure cloud based environment in which only the specified client can operate. This cloud referred to on-premise cloud used to provide the high level control over cloud services and infrastructure which is controlled or owned by an organization. It is built specifically to provide the services within an organization for maintaining the security and privacy [5]. Examples- HP data center.

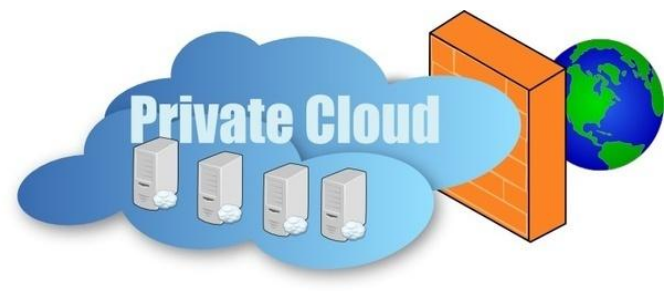


Vol. 8, Issue 4, April 2019

\section{Hybrid Cloud}

Any organization uses public and private cloud.it is combination of service provided by public cloud and private cloud. This cloud combines both public cloud and private models where cloud computing ecosystem is hosted and managed by third party (off-premise) but only an organization can privately use some dedicated resources [5]. This deployment cloud is a combination of two or more clouds (public, private or community). In this model, the privacy of the organization along with the availability for multi-tenancy with the ability to connect with other organizations is satisfied. Examples: Cybercon.com (US Microsoft Hybrid Cloud), and Bluemix.net (IBm Cloud App Development), etc.

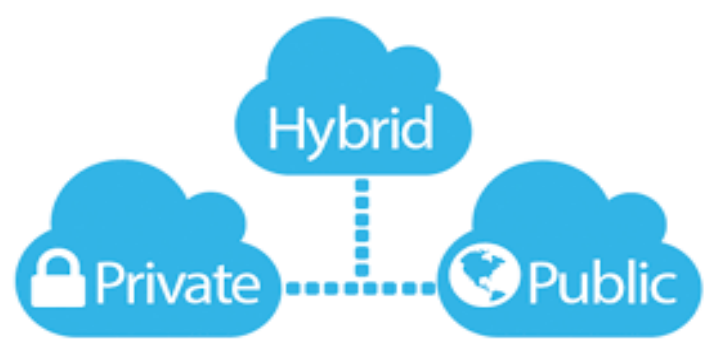

\section{Community Cloud}

This cloud allows the cloud computing environment can be shared or managed by a number of related organizations. This deployment cloud is operated by a community of group of organizations which share common concerns such as storage or security. These clouds can also be hosted and managed, both internally and externally. This model of cloud follows multi-tenant infrastructure. Some of the community clouds are Salesforce and QTS DataCenters.

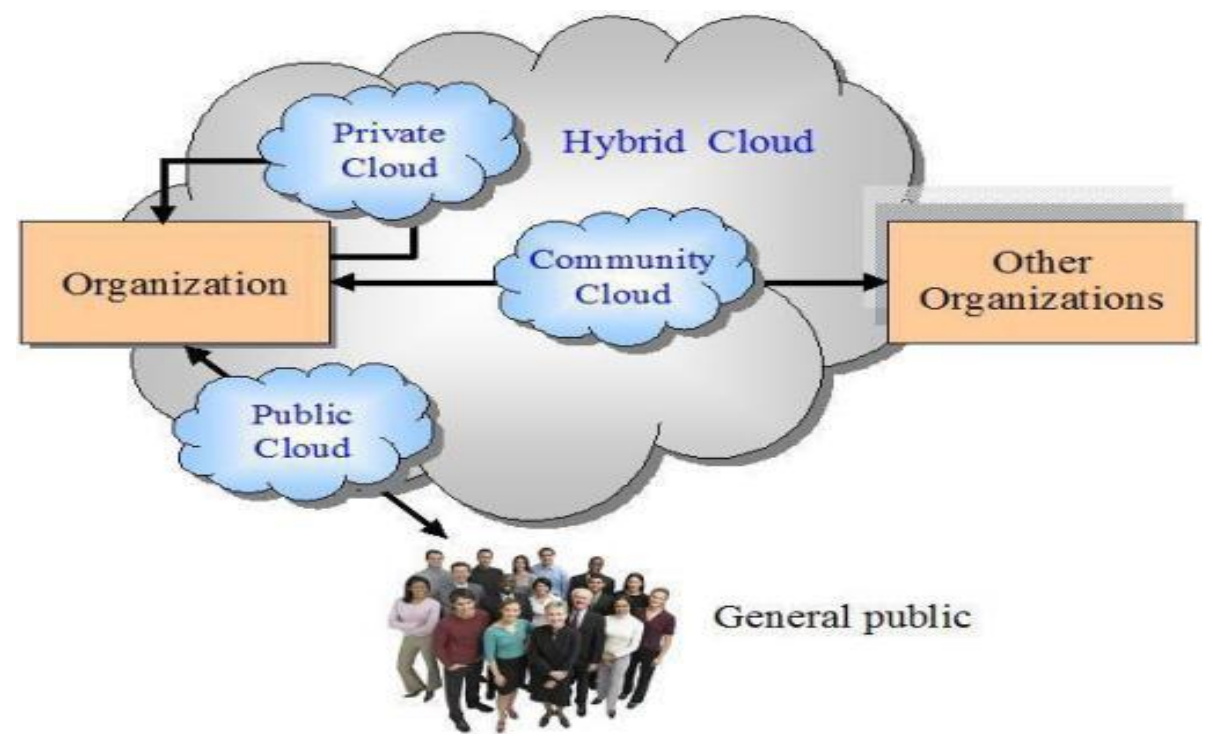

\section{CHALLENGES OF CLOUD COMPUTING}

Despite the potential gains achieved from the cloud computing, the organizations are slow in accepting it due to the following limitations: data loss, data cleaning, account hijacking, less control over the process, insider attacks by the CSP's, lack of legal aspects, lack of portability/migration from one service provider to another, less reliable, lack of auditability, less QoS .These limitations lead to the issues or challenges such as -security, interoperability, virtualization, data leakage, resource sharing, load balancing ,multi-tenancy, and Service Level Agreement (SLA)[6]. There are many benefits as mentioned above, even though cloud computing has many challenges. While moving from owning site to cloud space, companies must aware about the benefits and challenges of cloud computing. While analyzing these challenges, security of data is the most tedious work in cloud computing. According to a survey carried out by Gartner more than $70 \%$ of Chief Technical Officers believed that the primary reason for not using cloud computing services is that of the data security and privacy concerns. Convincing the organizations especially small ones about security concern is a tedious work; they are not ready to throw away their infrastructure and immediate move to cloud. Most of the organizations are closely watching this issue and not ready to shift to cloud space, this is main reason in the lack of maturity any other unauthorized user sharing the application or platform in the cloud, this cause the 
integrity failure. As data are the base for providing cloud computing services, such as Data as a Service, Software as a Service, Platform as a Service, keeping data integrity is a fundamental task.

\section{Cloud Challenges}

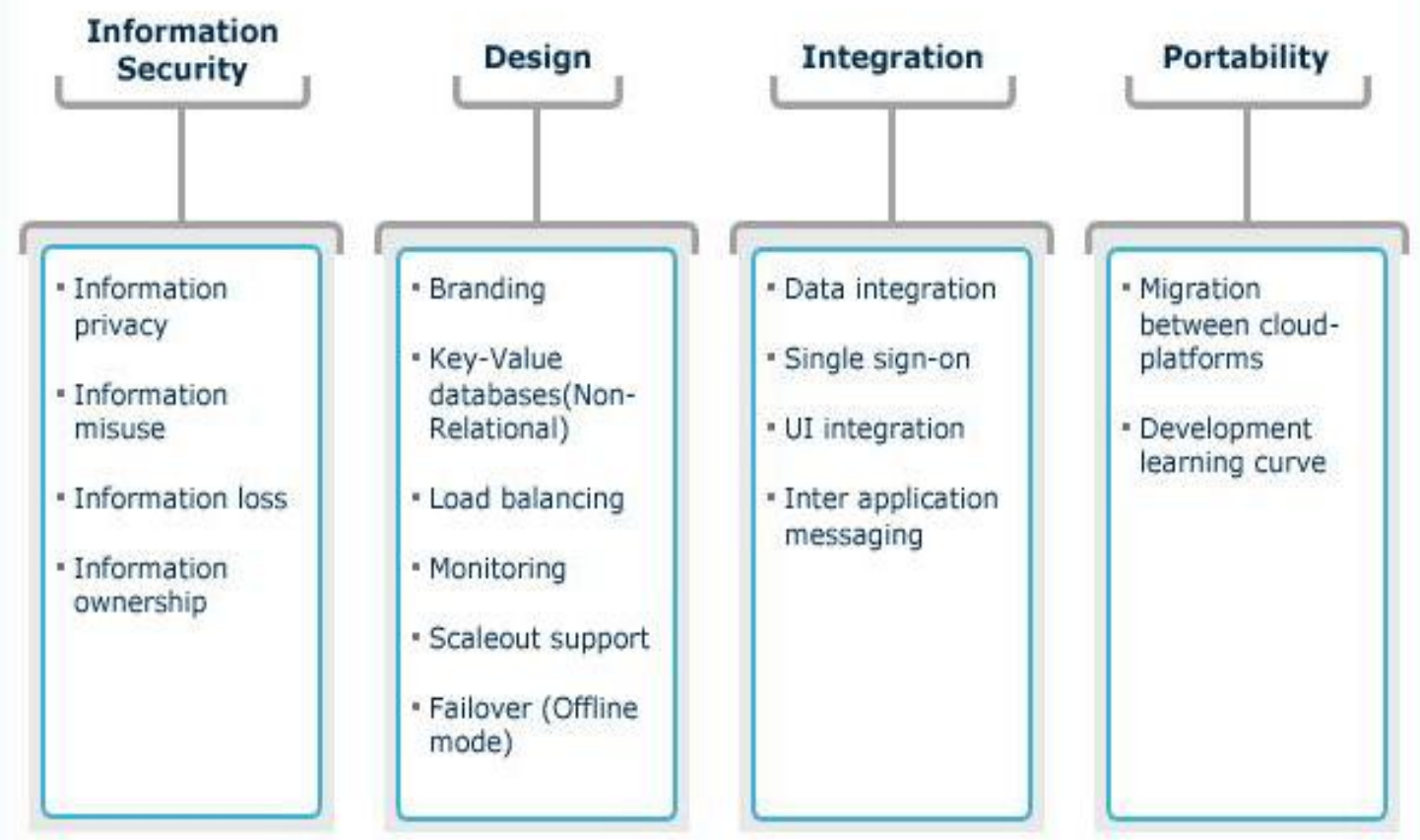

VI. DATA RECOVERY TECHNIQUE IN CLOUD COMPUTING

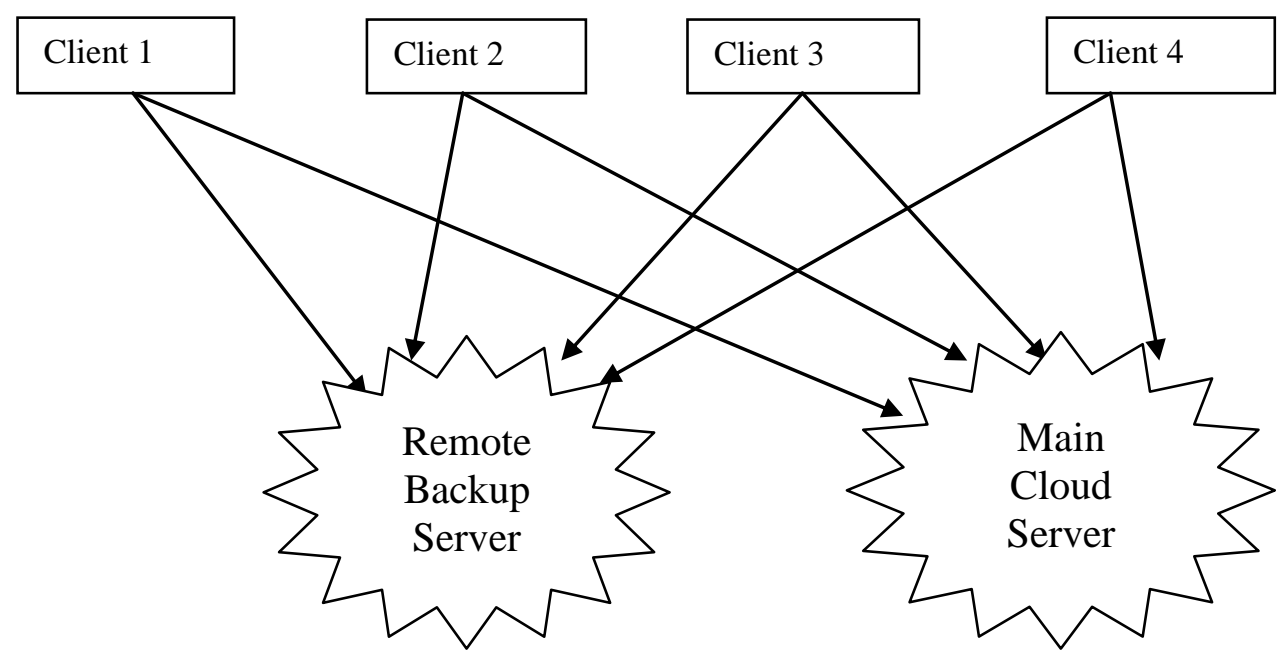

The above architecture shows three modules such as Remote Backup Server, Main Cloud Server and Number of Clients/users. Remote Backup server maintains the replicated copies of main server and is called as remote repository. The main server is called as central repository it stores all the user data. The user uploads the file to main cloud server; the main cloud server stores all the data in backup server. If user wants to retrieve the file from cloud then file is searched in main cloud server firstly, if the data is not present in main server then it is checks in backup server to retrieve lost data. The loss of data or data crash happens due to natural disasters or human made disasters in main server. To recover the lost data a recovery technique is essential. The replicated copies of data are maintained in more than one server to recover data. When data loss occurs at one location it can be retrieved from other backup server using 'Enriched Genetic Algorithm' (EGA). 


\section{A. Procedure of EGA}

1. User uploads the file $\mathrm{F}$ to the $\mathrm{N}$ cloud servers.

2. From the file $\mathrm{F}$ generates hash code $\mathrm{H} 1$ and stored in data base.

3. Calculate the size of file.

4. User has to select the file to be downloaded.

5. If the file is deleted then it is retrieved from the backup Server

6. Select the file to be downloaded and generate the hash code $\mathrm{H} 2$.

7. If both the hash codes are same then we retrieved the original file.

\section{B. Data Recovering Algorithm}

1. User has to select the file to be downloaded.

2. From the transaction table get the numbers of cloud storage containing the file and $\mathrm{N}$ server configuration details.

3. $\mathrm{I}=1$

4. Select Ith server status, if status is Deactivate then go to step 9.

5. Download file from Ith server

6. Generate hash code $\mathrm{H} 2$ from the file.

7. Fetch the hash code from Data base.

8. If $\mathrm{H} 1=\mathrm{H} 2$ the go to step 12 .

9. $\mathrm{I}=\mathrm{I}+1$

10. IF $\mathrm{I}<=\mathrm{N}$ go to step 12 .

11. Display "File recovered Successful": STOP.

12. Display "File Integrity check is successful": STOP.

13. Display "File Integrity check is not successful": STOP

When user request the file from main cloud server he has to select the file from the Data Recovery Application then the data Recovery Server will select the corresponding cloud sever details from the Database and also it will check for the cloud availability for recovering the file, if cloud server is not available then it will be recovered from another cloud server. While recovering it will generate the HASH Key (H2), then it will check for the H1 \& $\mathrm{H} 2$ for the integrity check. Finally the file is recovered from the backup server.

\section{CONCLUSION}

Cloud computing is an Internet-based computing service provided by the third party allowing share of resources and data among devices. It is widely used in many organizations nowadays and becoming more popular because it changes the way of how the Information Technology (IT) of an organization is organized and managed. It provides lots of benefits such as simplicity and lower costs, almost unlimited storage, least maintenance, easy utilization, backup and recovery, continuous availability, quality of service, automated software integration, scalability, flexibility and reliability, easy access to information, elasticity, quick deployment and lower barrier to entry. In this new era the use of cloud computing service is increasing rapidly, but the security issues of the cloud computing became a challenge. Cloud computing must be safe and secure enough to ensure the privacy of the users. In this paper firstly we have illustrated the architecture of the cloud computing, then discuss the most common security issues of using cloud and then we have proposed a data recovery algorithm for cloud computing environment.

\section{REFERENCES}

[1]. Vivek Paul, Supriya Pandita, Prof. Meera Randiva," Cloud computing review," International Research Journal of Engineering and Technology (IRJET), Volume: 05 Issue: 03, pp. 1454-1456, Mar.2018.

[2]. Prof. Syed Neha Samreen, Prof. Neha Khatri-Valmik, Prof. Supriya Madhukar Salve, Mr. Pathan Nouman Khan ," research to cloud computing ," International Research Journal of Engineering and Technology (IRJET), Volume: 05 Issue: 02, pp.785-788 , Feb.2018.

[3]. Priyanshu Srivastava, Rizwan Khan ," A Review Paper on Cloud Computing ," International Journals of Advanced Research in Computer Science and Software Engineering ISSN: 2277-128X,Volume-8, Issue-6, pp .17-20, june. 2018

[4]. Dr. Chinthagunta Mukundha ,K. Vidyamadhuri ," Cloud Computing Models : A Survey," Advances in Computational Sciences and Technology ISSN 0973-6107, pp. 747-761, Volume 10 , Number 5 .(2017).

[5]. Mahantesh N. Birje, Praveen S. Challagidad, R.H. Goudar, Manisha T. Tapale ,"Cloud computing review: concepts, technology, challenges and security" Int. J. Cloud Computing, Vol. 6, No. 1,pp.32-57,January 2017.

[6]. Nasarul Islam.K.V, "Review on Benefits and Security Challenges of Cloud Computing", International Journal of Computer Science and Information Technologies, Vol. 8 (2), pp.224-228, 2017.

[7]. Sanjana Sharma, Sonika Soni, Swati Sengar ," Security in Cloud Computing," National Conference on Security Issues in Network Technologies (NCSI), pp.01-06, August 11-12,2012.

[8]. L. Ertaul, S. Singhal, and G. Saldamli," Security Challenges in Cloud Computing” https://www.researchgate.net/publication/267697749, 22 December 2014. 
[9]. Dr. Amit Agarwal, Saloni Jain ," Efficient Optimal Algorithm of Task Scheduling in Cloud Computing Environment ," International Journal of Computer Trends and Technology (IJCTT) ,volume 9 number , pp.344-3497, 7Mar 2014

[10]. Ajay Kumar Bharti, Neha Verma, Deepak Kumar Verma, "A Review on Big Data Analytics Tools in Context with Scalability", International Journal of Computer Sciences \& Engineering, Vol.7, Issue.2, pp.273-277, 2019. DOI: https://doi.org/10.26438/ijcse/v7i2.273277

[11]. Praveen S. Challagidad, Ambika S. Dalawai, Mahantesh N. Birje, "efficient and reliable data recovery technique in cloud computing" http://www.sciencepublishinggroup.com/j/iotcc,vol5,no.5, pp. 13-18, August 25, 2017.

[12]. Choubey R, Dubey R and Bhattacharjee, "A survey on cloud computing security challenges and threats" International Journal on Computer Science and Engineering (IJCSE) 33 1227-1231, 2011.

[13]. Kuyoro S O, Ibikunie F and Awodele O, "Cloud computing security issues and challenges", International Journal of Computer Networks (IJCN) 35 247-255, 2011.

\section{BIOGRAPHIES}

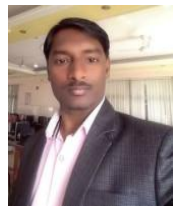

Dr. Deepak Kumar Verma, MCA, UGC-NET, Ph.D.(CS), pursed Bachelor of Science and Master of Computer Application from University of Lucknow, India in year 2011. Dr Verma completed his doctrate in Computer Science in the year 2016 and currently working with Department of Computer Science, Babasaheb Bhimrao Ambedkar University(A Central University), Lucknow, India. His research interests are Artificial intellegence, data security and Software Engineering. He has published number of research papers in reputed national/international journals and conferences.

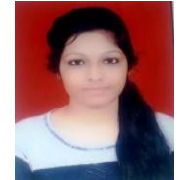

Ms. Tanya Sharma, pursed Bachelor of Computer Application (BCA) from MJP Rohilkhand University, Bareilly. She is currently pursuing Master of Computer Application (MCA) from Department of Computer Science, Babasaheb Bhimrao Ambedkar University (A Central University), Lucknow, India. Her Research Interests are Internet of Things, cloud computing and Big Data. 